\title{
Comparative Analysis of the Characteristics of Polar and Non-Polar Spiral Polarization Rotators
}

\author{
Andrey L. Sokolov \\ National Research University, "Moscow Power Engineering Institute” (MPEI), Moscow, Russia \\ Email: alsokolov@bk.ru
}

How to cite this paper: Sokolov, A.L. (2020) Comparative Analysis of the Characteristics of Polar and Non-Polar Spiral Polarization Rotators. Optics and Photonics Journal, 10, 13-27.

https://doi.org/10.4236/opj.2020.102002

Received: January 22, 2020

Accepted: February 25, 2020

Published: February 28, 2020

Copyright (๑) 2020 by author(s) and Scientific Research Publishing Inc. This work is licensed under the Creative Commons Attribution International License (CC BY 4.0).

http://creativecommons.org/licenses/by/4.0/

\section{(c) (i) Open Access}

\begin{abstract}
Spiral polarization rotators, rotating polarization ellipse axes clockwise or counterclockwise, depending on the azimuth angle in the transverse plane, are considered. It is shown that spiral polarization rotators lead to a change in the order of optical vortices with circular polarization. A comparative analysis of spiral rotators of two types (polar and non-polar) is carried out, using a mirror that allows light to pass in the opposite direction through the rotator. The effect of spiral rotators on optical vortices in a resonator is studied. It is shown that spiral rotators can preserve or accumulate changes of the vortex order during the passage of the beam in both directions. The properties of the spiral rotator and the cube-corner reflector with a special phase-correcting coating, as a diffractive polarization-optical element, are compared.
\end{abstract}

\section{Keywords}

Polarization-Symmetric Structures, Optical Vortex, Circular Polarization, Spiral Polarization Rotator, Cube-Corner Reflector

\section{Introduction}

Study of polarization-inhomogeneous beams allows to conclude that, despite the complex structure, such beams have certain types of polarization symmetry [1]-[6]. Polarization structures with axial symmetry are characterized by the following: in the cross-sectional plane a certain orientation of the polarization ellipse (polarization azimuth $\psi$ ) is a constant along the radius vector, outgoing from the beam axis, but changes in proportion to coordinate azimuth $\varphi$. After a complete rotation of the radius vector (returning to the initial value of the azimuth) the axis of the polarization ellipse makes $s$ full turns. 
The beams with axisymmetric polarization structure have two modifications, depending on rotation direction of the polarization ellipse axis and are closely related to optical vortices.

Currently, variety of data on optical vortices [7] [8] has been accumulated: production methods, transformation, analysis are considered in [9] [10] [11] and others works, diversified bibliography on this topic is given in [12]. At the same time, mainly amplitude-phase characteristics of axisymmetric beams and optical vortices are discussed [13] [14] [15].

Much less attention is paid to polarization characteristics and diffraction polarization-optical devices [16] [17] [18]. For example, sharp focusing is applied to identify the polarization state [12]. In [16] new polarization convertor, based on diffractive optical elements, is proposed. The corner-cube reflector with special faces coating as new diffraction polarization-optical devices is considered in [17] [19].

The aim of this work is to study spiral polarization rotators, which-like conventional polarization rotators-exist in two forms: polar and non-polar, and their influence on optical vortices, including in optical resonators. This article is a continuation of [19], where, along with polarization-symmetric structures of optical vortices, spiral polarization elements were considered, except for spiral rotators. In this work, the difference between the properties of polar and non-polar spiral polarization rotators is analyzed.

\section{Polarization-Symmetric Structures and Their Correlation with Optical Vortices}

Polarization-symmetric structures with a regular change of polarization in a beam cross section will be denoted by capital letters indicating the direction of rotation of the semi-major axis of the polarization ellipse: $P$ (counterclockwise) and $N$ (clockwise) when viewed towards the ray direction [19].

Orthogonal polarization structures of $P$-modification in Cartesian basis are described by Jones vectors of the following type:

$$
\boldsymbol{D}_{P r}=\left(\begin{array}{c}
\cos (s \varphi) \\
\sin (s \varphi)
\end{array}\right), \boldsymbol{D}_{P a}=\left(\begin{array}{c}
-\sin (s \varphi) \\
\cos (s \varphi)
\end{array}\right) .
$$

Here indices $r$ and a denote orthogonal structures: radially polarized and azimuthally polarized, respectively. For these structures, the plane of vector $E$ oscillations makes $s$ counterclockwise rotations in the transverse beam plane when observed towards the beamand axisymmetric polarization structure order.

Polarization structures of $\mathrm{N}$-modification with the rotation of the plane of vector $E$ oscillations in a clockwise direction are described by Jones vectors of the following type:

$$
\boldsymbol{D}_{N r}=\left(\begin{array}{c}
\cos (s \varphi) \\
-\sin (s \varphi)
\end{array}\right), \boldsymbol{D}_{\mathrm{Na}}=\left(\begin{array}{c}
\sin (s \varphi) \\
\cos (s \varphi)
\end{array}\right) .
$$


An optical vortex with an axisymmetric polarization structure has a spiral phase structure alongside with the axial polarization symmetry with srepetitions of the polarization state in the cross section (in particular, this is the rotation of polarization plane). Optical vortices with an axisymmetric polarization structure of various types vary depending on the number of repetitions of a phase- $q$ (the optical vortex order) and polarization state $-s$ (the order of axisymmetric polarization structure).

The general expression for Jones vector of an optical vortex with axisymmetric polarization structure of $P$-modification in Cartesian basis is:

$$
\boldsymbol{D}_{P}=\left[\left(\begin{array}{c}
\cos (s \varphi) \\
\sin (s \varphi)
\end{array}\right)+\Gamma_{P}\left(\begin{array}{c}
-\sin (s \varphi) \\
\cos (s \varphi)
\end{array}\right)\right] \exp ( \pm i q \varphi),
$$

and for $N$-modification:

$$
\boldsymbol{D}_{N}=\left[\left(\begin{array}{c}
\cos (s \varphi) \\
-\sin (s \varphi)
\end{array}\right)+\Gamma_{N}\left(\begin{array}{c}
\sin (s \varphi) \\
\cos (s \varphi)
\end{array}\right)\right] \exp ( \pm i q \varphi),
$$

where $\Gamma_{P}, \Gamma_{N}$-polarization variables, characterizing polarization state.

In particular case, if $\Gamma_{P}= \pm i, \Gamma_{N}= \pm i$ and $s=1, q=0$ expressions (3), (4) describe optical vortices with circular polarization:

$$
\begin{aligned}
& \boldsymbol{D}_{r L}=\left[\left(\begin{array}{c}
\cos \varphi \\
\sin \varphi
\end{array}\right)+i\left(\begin{array}{c}
-\sin \varphi \\
\cos \varphi
\end{array}\right)\right]=\left(\begin{array}{c}
1 \\
i
\end{array}\right) \exp (-i \varphi) \\
& \boldsymbol{D}_{I R}=\left[\left(\begin{array}{c}
\cos \varphi \\
\sin \varphi
\end{array}\right)-i\left(\begin{array}{c}
-\sin \varphi \\
\cos \varphi
\end{array}\right)\right]=\left(\begin{array}{c}
1 \\
-i
\end{array}\right) \exp (i \varphi) \\
& \boldsymbol{D}_{r R}=\left[\left(\begin{array}{c}
\cos \varphi \\
-\sin \varphi
\end{array}\right)+i\left(\begin{array}{c}
\sin \varphi \\
\cos \varphi
\end{array}\right)\right]=\left(\begin{array}{c}
1 \\
i
\end{array}\right) \exp (i \varphi) \\
& \boldsymbol{D}_{l L}=\left[\left(\begin{array}{c}
\cos \varphi \\
-\sin \varphi
\end{array}\right)-i\left(\begin{array}{c}
\sin \varphi \\
\cos \varphi
\end{array}\right)\right]=\left(\begin{array}{c}
1 \\
-i
\end{array}\right) \exp (-i \varphi)
\end{aligned}
$$

Here the first index designates the type of circular polarization: right circular (if vector $E$ rotates clockwise when observed towards the beam) $-r$ and left circular (if vector $E$ rotates counter-clockwise) $-l$; the second index with capital letters designates optical vortex type: right-handed $(R)$ and left-handed $(L)$.

Such polarization optical vortices have optical angular moment $2,-2$ and 0 , according to [20] [21] [22].

Optical vortex with arbitrary polarization can be expressed by superposition of two beams with axisymmetric structure of the same order.

\section{Reflection from a Mirror}

Jones matrix of a high-quality mirror for a plane polarization-homogeneous wave is given by:

$$
M_{P}=\left(\begin{array}{cc}
1 & 0 \\
0 & -1
\end{array}\right)
$$


In case of polarization-inhomogeneous wave transformation of vector $E$ defined by (6) is preserved for each cross-section point if wave front curvature coincides with mirror curvature or their difference can be neglected.

If coordinates of a light beam point are determined in cylindrical coordinate system by an azimuthal angle $\varphi$ and transverse radius $r$, then it should be taken into account that for the reflected wave $\varphi_{\text {refl }}=\pi-\varphi_{\text {inc }}$. Suchwise, polarization optical vortices (5) change the rotation sign and vortex sign. For instance, if

$$
\boldsymbol{D}^{(i n c)}=\boldsymbol{D}_{r R}=\left(\begin{array}{l}
1 \\
i
\end{array}\right) \exp (i q \varphi)
$$

then

$$
\boldsymbol{D}^{(r e f l)}=\boldsymbol{D}_{l L}=\left(\begin{array}{c}
1 \\
-i
\end{array}\right) \exp (-i q \varphi)
$$

and

$$
\boldsymbol{D}^{(i n c)}=\boldsymbol{D}_{r L}=\left(\begin{array}{l}
1 \\
i
\end{array}\right) \exp (-i q \varphi)
$$

transforms to

$$
\boldsymbol{D}^{(\text {refl })}=\boldsymbol{D}_{l R}=\left(\begin{array}{c}
1 \\
-i
\end{array}\right) \exp (+i q \varphi) .
$$

High-quality mirror does not disturb polarization-optical symmetry of beams, because transformations $r R \Leftrightarrow l L$ and $r L \Leftrightarrow l R$ are realized. Obviously, optical angular moment magnitude is preserved.

\section{Spiral Rotators (SR)}

Transformation of polarization-symmetric structures, in particular, change of the direction of rotation of the plane of $\boldsymbol{E}$ oscillations in the transverse plane, as well as transformation of optical vortices with axisymmetric polarization structure, is possible by means of special polarization-inhomogeneous devices, spiral polarization rotator (SR) being among them.

Jones matrices of spiral rotator of $u$ order are given by:

$$
M_{P}=\left(\begin{array}{cc}
\cos (u \varphi) & -\sin (u \varphi) \\
+\sin (u \varphi) & \cos (u \varphi)
\end{array}\right), M_{N}=\left(\begin{array}{cc}
\cos (u \varphi) & +\sin (u \varphi) \\
-\sin (u \varphi) & \cos (u \varphi)
\end{array}\right) .
$$

According to our definition, a positive $\mathrm{SR}_{\mathrm{P}}$ rotates the polarization ellipse axes counterclockwise by a value depending on azimuth $\varphi$, in other words, it twists the polarization structure counterclockwise when it is observed towards the beam. Negative rotator $\mathrm{SR}_{\mathrm{N}}$ twists polarization structure clockwise.

Spiral rotators are characterized by the presence of semi-axis of symmetry, from which azimuth should be counted. For points of a transverse plane, superadjacent this semi-axis SR, the state of polarization of an incident light beam is preserved. Thus, unlike standard polarization-uniform rotators, spiral rotators are not invariant to the rotation of the Cartesian polarization basis.

When a linearly polarized wave propagates a $\mathrm{SR}_{\mathrm{p}}, P$-modifiedbeams are gen- 
erated, in particular, if $u=1$, radial polarized beam is generated in case of horizontal polarization, and azimuthal polarized beamoccurs in case of vertical polarization. Negative rotator $\mathrm{SR}_{\mathrm{N}}$ forms $N$-modified beams from linear polarization.

SR affects a beam with $P$-modified axisymmetric polarization structure, changing its order: the new order $s^{\prime}$ is related to the original order $s$ and the order of the spatial rotator $u$ by the following relation: $s^{\prime}=u+s$. For a beam with $N$-modified axisymmetric polarization structure we have $s^{\prime}=u-s$. Spatial rotation sign should be taken into account in these expressions. Thus, if signs of SR and of modification of axisymmetric polarization structure coincide, its order increases; if signs differ, the order decreases.

Let us consider the effect of a spiral rotator on optical vortices with circular polarization. The order of an optical vortex can either increase or decrease. This is determined by the ratio of the signs of SR and of ellipticity angle $\chi$ (right $(+)$ or left $(-)$ rotation), while circular polarization sign does not change:

$$
\begin{aligned}
& \left(\begin{array}{cc}
\cos (u \varphi) & \pm \sin (u \varphi) \\
\mp \sin (u \varphi) & \cos (u \phi)
\end{array}\right)\left(\begin{array}{c}
1 \\
i
\end{array}\right) \exp (-i q \varphi)=\left(\begin{array}{c}
1 \\
i
\end{array}\right) \exp [-i(q \mp u) \varphi] \\
& \left(\begin{array}{cc}
\cos (u \varphi) & \pm \sin (u \varphi) \\
\mp \sin (u \varphi) & \cos (u \varphi)
\end{array}\right)\left(\begin{array}{c}
1 \\
-i
\end{array}\right) \exp (-i q \varphi)=\left(\begin{array}{c}
1 \\
-i
\end{array}\right) \exp [-i(q \pm u) \varphi] .
\end{aligned}
$$

In accordance with our terminology positive SR destroys vertices with Jones vectors $\boldsymbol{D}_{r R}$ and $\boldsymbol{D}_{I L}$ (decreases the order) and twists vertices $\boldsymbol{D}_{I R}$ and $\boldsymbol{D}_{r L}$ (increases the order). On the contrary, negative SR destroys vortices $\boldsymbol{D}_{I R}$ and $\boldsymbol{D}_{r L}$ and twists vortices $\boldsymbol{D}_{r R}$ and $\boldsymbol{D}_{l L}$.

Let us consider circular polarized optical vortex after its spiral phase structure has been destroyed by a spiral rotator. Let the optical vortex be a combination of two axisymmetric beams. In particular, as is well known, the left optical vortex is a superposition of radially polarized and azimuthally polarized beams shifted in phase by $90^{\circ}$. After the destruction of the phase vortex structure, each of these beams becomes linearly polarized in mutually perpendicular planes with a phase shift of $90^{\circ}$, which forms circular polarization. Alongside the spatial amplitude distribution is still corresponding to axisymmetric beams with zero intensity on the axis. Due to diffraction, such beams do not retain their amplitude-phase distribution in the far zone: the beam energy "flows" from the periphery to the center.

\section{Polar and Non-Polar Spiral Rotators}

Like polarization-uniform rotators, spiral rotators are divided into polar, magneto-optical or Faradaytype (further they are designated as SRF) and non-polar, based on natural optical activity, type (SRO).

In the coordinate system, which remains right for the reversewave, the sign of the polar SRF (positive or negative) is reversed, and the sign of the non-polar SRO is retained. However, unlike common Jones matrices in this case azimuth change $\varphi_{\text {out }}=\pi-\varphi_{\text {in }}$ should be taken into account. As a result, if $u=1$, for non-polar positive SRO we have: 


$$
\begin{gathered}
M_{P O}^{(+)}=\left(\begin{array}{cc}
\cos \varphi & -\sin \varphi \\
+\sin \varphi & \cos \varphi
\end{array}\right), \\
M_{P O}^{(-)}=\left(\begin{array}{cc}
\cos (\pi-\varphi) & -\sin (\pi-\varphi) \\
+\sin (\pi-\varphi) & \cos (\pi-\varphi)
\end{array}\right)=-\left(\begin{array}{cc}
\cos \varphi & +\sin \varphi \\
-\sin \varphi & \cos \varphi
\end{array}\right) .
\end{gathered}
$$

Here superscript indicates direct $(+)$ and reverse $(-)$ waves.

Thus, unlike usual Jones matrix in natural-optical rotator, in this case the sign of the rotator in Jones matrix has changed to the opposite.

Consequently, for polar (Faraday) SRF we have:

$$
M_{P F}^{(+)}=\left(\begin{array}{cc}
\cos \varphi & -\sin \varphi \\
+\sin \varphi & \cos \varphi
\end{array}\right), M_{P F}^{(-)}=\left(\begin{array}{cc}
\cos \varphi & -\sin \varphi \\
+\sin \varphi & \cos \varphi
\end{array}\right) .
$$

Mechanism of the sign change remains for negative SR.

Combined affection of spiral rotators and high-quality mirror onto polarization optical vortices is illustrated by Figure 1 and Figure 2 .

Analyzing the figures one would easily notice the difference in action of Faraday spiral rotator and action of rotator, based on natural optical activity.
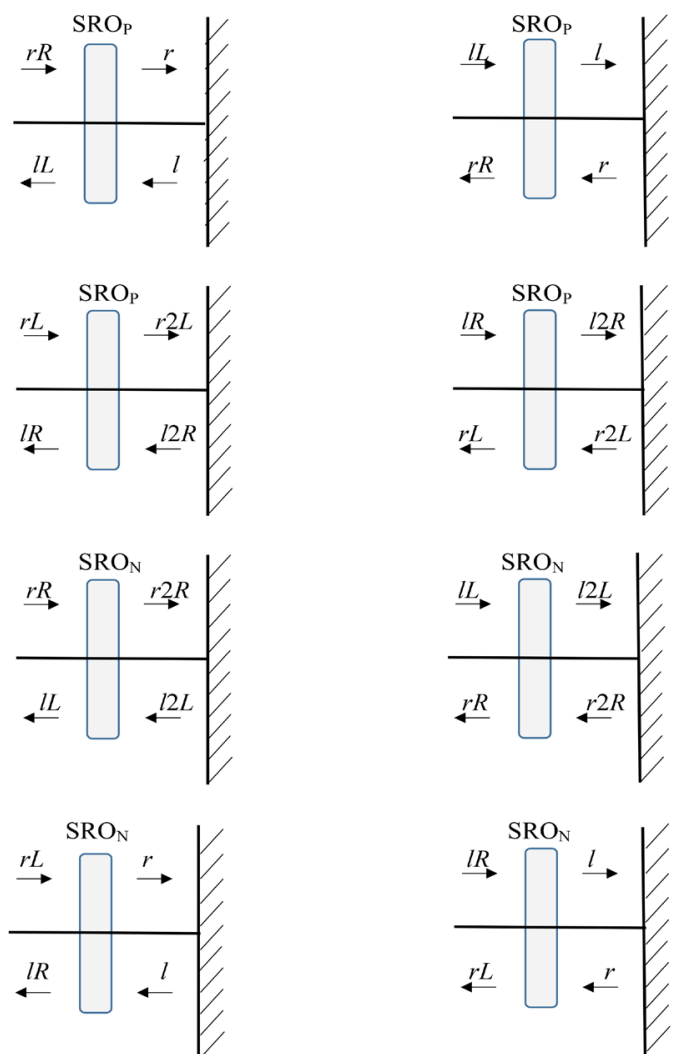

Figure 1. Passage of polarization optical vortices through natural spiral rotators with different signs, where: $r$-right circular polarization, $l$-left circular polarization, $R$-right (positive) vortex, $L$-left (negative) vortex. The combination of letters indicates the combination of the corresponding polarization state and the vortex sign. $S R O_{P}$ - positive natural optical spiral rotator, $S R O_{N}$ negative natural optical spiral rotator. 

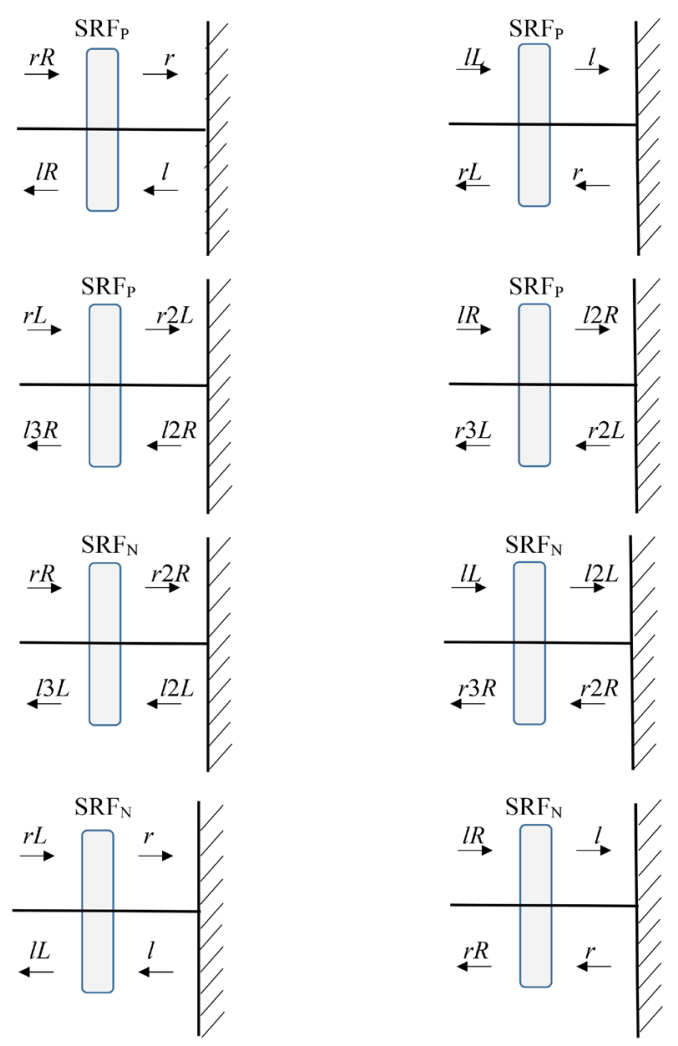

Figure 2. Passage of polarization optical vortices through Faraday spiral rotators with different signs, where: $r$-right circular polarization, $l$-left circular polarization, $R$-right (positive) vortex, $L$-left (negative) vortex. The combination of letters indicates the combination of the corresponding polarization state and the vortex sign. $S R F_{P}$ - Positive Faraday Spiral Rotator, $S R F_{N}-$ Negative Faraday Spiral Rotator.

If the sign of the optical vortex is opposite to the sign of circular polarization, then after passing twice the positive Faraday spiral rotator in the direct and reverse direction, the order of the optical vortex increases by $2: r L \Leftrightarrow 3 I R$ and $I R \Leftrightarrow r 3 L$.

However, if the sign of vortex coincides with the sign of circular polarization, vortices will only change the sign: $r R \Leftrightarrow I L$ and $r L \Leftrightarrow I R$. It is correlated with the abovementioned fact that positive SR destroys vortices (reduces order) $r R$, $l L$ and twists vortices (increases order) $r L$ and $l R$. For negative Faraday spiral rotator SRF the situation is reverse: the vortex order grows for vortices with coincided sign of circular polarization and vortex.

In case of both positive and negative SRO, based on natural optical activity, only change of optical vortex sign takes place.

Circular polarization sign changes to the opposite in all cases.

The main difference between the effect of spiral rotator, based on natural optical activity, and a magneto-optical one, combined with a plane mirror, is that the first type does not disturb the polarization-wave symmetry of a beam, while the second one disturbs, and orbital moment of a transmitted beam is not preserved. 


\section{Spiral Rotators in Linear and Ring Resonators}

Let's perform a thought experiment: a polarization optical vortex passes through a linear two-mirror resonator; a beam splitting cube, which ideally does not change the polarization state, is used to enter the resonator (Figure 3). Spiral rotator based on natural optical activity SRO and Faraday spiral rotator SRF are placed in turns inside the resonator.

As follows from the analysis of Figure 3, there is a significant difference in the form of a beam exiting the resonator in these two examples. In the first case the light, passing through the spiral rotator based on natural optical activity, restores its polarization state and vortex order. In the second case the beam form changes significantly: both change of vortex sign and increase of its order are possible, in other words, the polarization-wave structure of a beam and orbital moment change.

In a four-mirror ring resonator a wave increases optical vortex order after each passage, respectively, its transverse size and orbital angular moment increase. It is obvious that increase of the order corresponds to an increase of diffraction losses on the diaphragms. One can assume that order of optical vortex, generated in the ring resonator, is approximately equal to Fresnel number: $a^{2} / \lambda L$, where $\alpha$-diaphragm aperturesize, $L$-resonator length, $\lambda$-wavelength.

As it is known, the so-called Faraday cells, consisting of two crossed quarter-wave plates and a magneto-optical rotator between them, are used in laser gyroscopes to produce a phase shift between the passing counter-propagating waves. For this effect, it is necessary that the directions of rotation of the vector $E$ of counter-propagating waves be opposite with respect to the magnetic field $H$ of the Faraday rotator. The maximum effect is achieved in the case of circular polarizations. It should be emphasized that if the counter-waves have right and left circular polarization in their own bases, i.e. relative to the vector $H$ the vectors $E$ of the counter-propagating waves rotate in the same direction, then the phase shift is absent.

We show that in the case of spiral Faraday rotators this requirement is optional. This statement is illustrated in Figure 4, where two positive SRF are shown for symmetry. Counter waves at the entrance from both sides have opposite sign circular polarizations and the same sign and order of the optical vortex. A wave propagating from left to right only changes the vortex sign, and a wave propagating from right to left increases the order of the vortex, and, therefore, the orbital optical moment and the order of the modes forming the vortex. As a result, the counter-propagating waves acquire a phase shift

$$
\Delta \varphi=\left(s_{1}-s_{2}\right) \arctan \left[\frac{\lambda d}{\pi w^{2}(1+\rho d)}\right],
$$

where $w$ is the parameter of the Gaussian mode distribution (radius with respect to the amplitude decrease in $e$ times); $\rho$-curvature of the wave front; $d$-the distance between two spiral Faraday rotators, whose thickness is neglected; $s_{1,2}$-polarization orders of the counter-propagating optical vortices. 


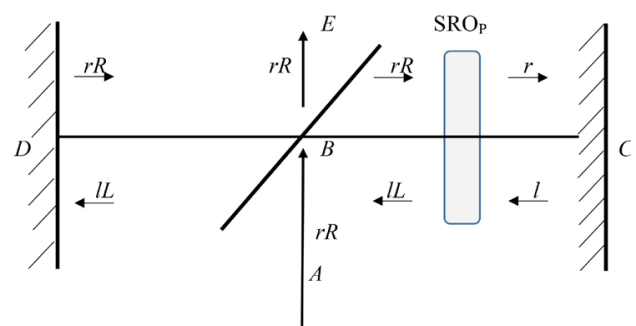

(a)

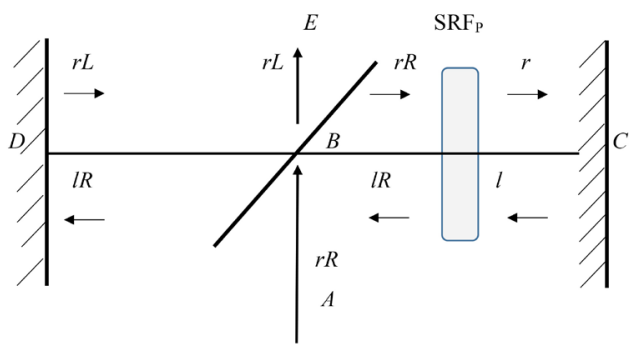

(b)

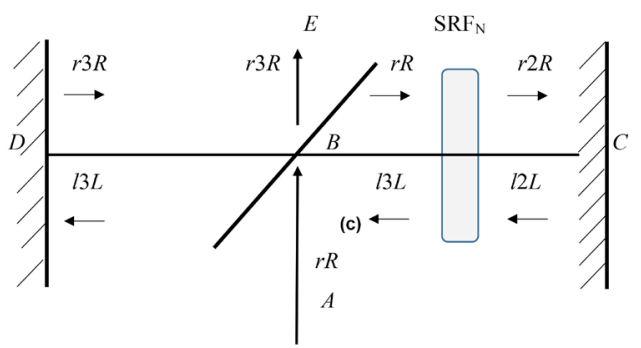

(c)

Figure 3. Passage of polarization optical vortices through natural (a) and Faraday (b) (c) spiral rotators, located in linear resonator. Incident light has right circular polarization. In case of a natural spiral rotator, right optical vortex with the right circular polarization remains at the output; in case of a negative spiral Faraday rotator, the sign of the optical vortex changes (b) or a third-order optical vortex appears (c).

$\mathrm{SRF}_{\mathrm{P}} \quad \mathrm{SRF}_{\mathrm{P}}$

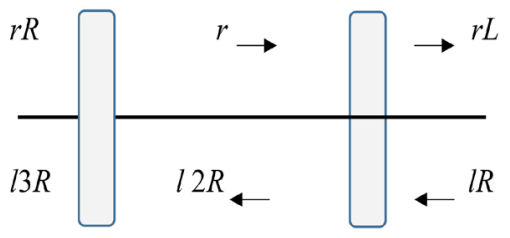

Figure 4. A device producing a phase shift (non-reciprocity) between counter-propagating waves, depending on the sign of the optical vortex. Optical vortices with right and left circular polarization pass through two spiral Faraday rotators, acquiring a different phase shift.

Note that this device can be used to identify the sign of an optical vortex, because the change of the waves sign leads to the absence of a phase shift. 


\section{Technical Implementation of Spiral Polarization Rotators}

Several ways of technical implementation of polarization rotators exist: medium with the natural optical rotation (crystals, solutions), magneto-optical medium under superimposed magnetic field, system, containing two expanded half-wave phase plates [23], nonplanar systems of reflectors, where beam reflects in orthogonal planes [24]. Diffraction optical elements, development and production of which is actively developed, have a special place. In particular, spiral phase plates, whose transmission function is proportional to $\exp (i q \varphi)$ [25] [26], are widely known. Spiral polarization rotators can be constructed based on these elements.

Application of crystalline spiral phase plates, giving different phase incursions for orthogonal linear polarization states, makes possible to obtain Jones matrix:

$$
M=\left(\begin{array}{cc}
\exp (i \varphi / 2) & 0 \\
0 & \exp (-i \varphi / 2)
\end{array}\right) .
$$

Such matrix in circular polarization basis takes form of a spiral polarization rotator:

$$
M_{c}=\left(\begin{array}{cc}
\cos \varphi & -\sin \varphi \\
+\sin \varphi & \cos \varphi
\end{array}\right) .
$$

SR affecting only linear polarization are easiest to be implemented. These plates have radial sectors, each of which has a half-wave phase plate with axes turned at a certain angle. For example, if there are six segments, these are the following angles: $0^{\circ},-120^{\circ},+120^{\circ}, 0^{\circ},-120^{\circ},+120^{\circ}$, counted counterclockwise when facing towards the beam.

Cube-corner reflector (CCR) [27] [28] with special phase-shifting coating [29] acts similarly. Depending on a phase shift of orthogonal components of vector $E$ occurring when reflected from three faces, different far-field diffraction patterns are formed. Figure 5 represents diffraction patterns and corresponding polarization distribution if incident light is circular polarized. With a phase shift equal to zero (or $180^{\circ}$, depending on coordinate axes orientation), CCR becomes kind of like a spiral polarization rotator.

In this case, Jones matrix at CCR output has form for different sectors (Figure 6) in the right coordinate system of the reflected wave:

$$
\begin{gathered}
M_{C C R}(5)=M_{C C R}(2)=-\left(\begin{array}{cc}
\cos (\pi / 3) & -\sin (\pi / 3) \\
+\sin (\pi / 3) & \cos (\pi / 3)
\end{array}\right), \\
M_{C C R}(6)=M_{C C R}(3)=-\left(\begin{array}{cc}
\cos (\pi / 3) & +\sin (\pi / 3) \\
-\sin (\pi / 3) & \cos (\pi / 3)
\end{array}\right), \\
M_{C C R}(4)=M_{C C R}(1)=\left(\begin{array}{ll}
1 & 0 \\
0 & 1
\end{array}\right) .
\end{gathered}
$$

In accordance with our terminology, such CCR is similar to negative SR of the second order.

Ideally, Jones matrix of the second order spiral reflector is: 


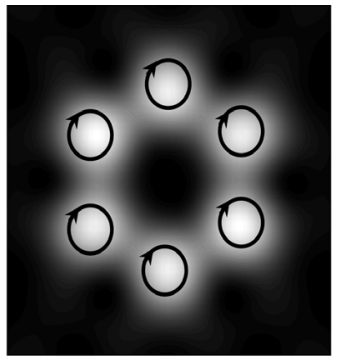

(a)

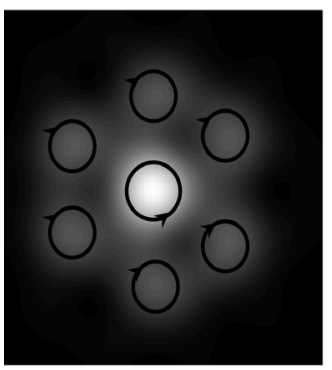

(c)

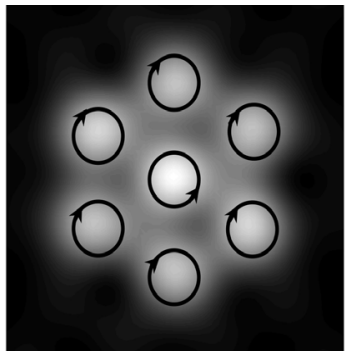

(b)

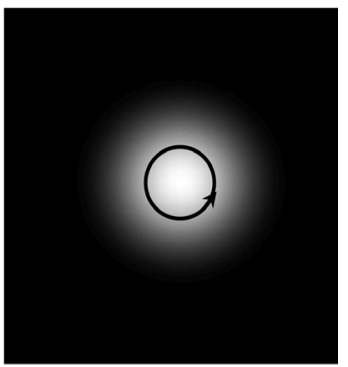

(d)

Figure 5. Far-field diffraction pattern and polarization in spots when right circular polarized light impinges onto a cub-corner reflector with different phase shift $\delta$ of vector $E$ components when reflected from the edges: $\delta=0^{\circ}$ (a), $\delta=$ $+30^{\circ}$ (b), $\delta=-40^{\circ}$ - corresponds to the total internal reflection (c), $\delta= \pm 170^{\circ}$ (d). If another coordinate frame is chosen, phase shift changes at $\pm 180^{\circ}$. In central spot polarization becomes orthogonal circular.

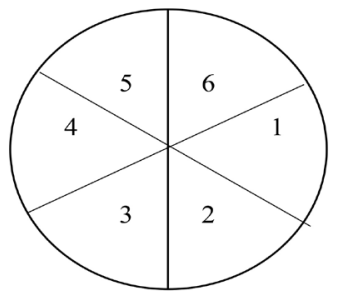

(a)

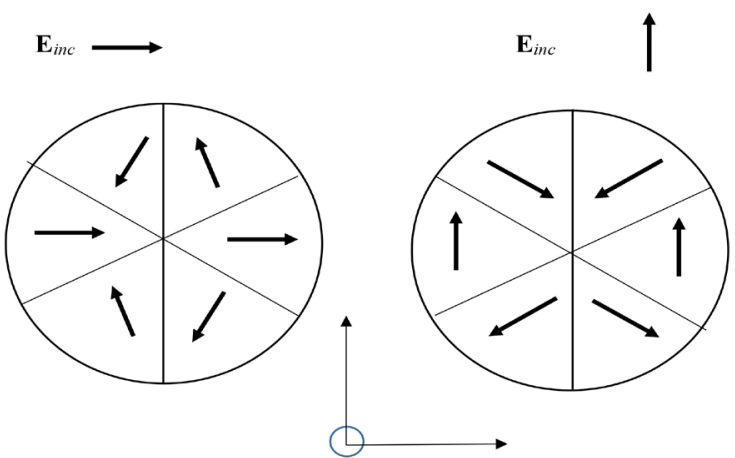

(b)

Figure 6. Polarization of light reflected from a cube-corner reflector with a special interference coating in the near zone. (a) Numbering of six segments of a cube-corner reflector; (b) Orientation vector $\boldsymbol{E}$ oscillations plane when incident light has orthogonal linear polarization. 


$$
M_{N}=\left(\begin{array}{cc}
\cos 2 \varphi & -\sin 2 \varphi \\
+\sin 2 \varphi & \cos 2 \varphi
\end{array}\right) .
$$

Worth noting is that technical implementation of SRO in the form of inhomogeneous sugar solution means that sugar concentration on the semi-axis of SRO is zero. In the case of an inhomogeneous magnetic field of the SRF, there is a semiaxis in the transverse plane along which the magnetic field vanishes or does not cause the rotation of the vector $E$. In contrast to these devices, in the case of the cube-corner reflector, the position of the semiaxis, for which there is no rotation, is not fixed in the transverse plane, but is determined by the orientation of the linear polarization of the incident wave.

\section{Conclusions}

The main results are the following:

1) Spiral polarization rotators are divided into two types: polar (magneto-optical) and non-polar (natural-optical), depending on whether their sign changes for the counter-propagating waves.

2) The structure of a circular polarized optical vortex is destroyed in a device, consisting of a polar (magnetic) spiral polarization rotator and a mirror, and optical orbital moment changes. A similar result occurs when spiral polarization rotator is placed into a linear resonator. Therefore, the polar spiral rotators can produces a phase shift (non-reciprocity) between counter-propagating optical vortices with circular polarization, depending on the sign of the optical vortex. For a non-polar spiral rotator, based on natural optical activity, this effect is not observed.

3) Cube-corner reflector with a special phase-correcting coating is a diffraction polarization-optical element with six segments and a kind of like a spiral rotator.

\section{Conflicts of Interest}

The author declares no conflicts of interest regarding the publication of this paper.

\section{References}

[1] Durnin, J., Miceli, J.J. and Eberly, J.H. (1987) Diffraction-Free Beams. Physical Review Letters, 58, 1499. https://doi.org/10.1103/PhysRevLett.58.1499

[2] Nesterov, A.V. and Niziev, V.G. (2000) Laser Beams with Axially Symmetric Polarization. Journal of Physics D: Applied Physics, 33, 1817-1822. https://doi.org/10.1088/0022-3727/33/15/310

[3] Nesterov, A.V., Niziev, V.G. and Sokolov, A.L. (2001) Transformative Problem for Radial-Polarization Radiation. Optics and Spectroscopy, 90, 1018-1022. https://doi.org/10.1134/1.1380793

[4] Dorn, R., Quabis, S. and Leuchs, G. (2005) Generation of a Radially Polarized Doughnut Mode of High Quality. Applied Physics B, 81, 597-600. https://doi.org/10.1007/s00340-005-1887-1

[5] Sokolov, A.L. (2008) Laser Beams with Regular Polarization Properties. Optics and Spectroscopy, 104, 138-139. https://doi.org/10.1134/S0030400X08010189 
[6] Zhan, Q. (2009) Cylindrical Vector Beams: From Mathematical Concepts to Applications. Advances in Optics and Photonics, 1, 1-57. https://doi.org/10.1364/AOP.1.000001

[7] Coullet, P., Gil, G. and Rocca, F. (1989) Optical Vortices. Optics Communications, 73, 403-408. https://doi.org/10.1016/0030-4018(89)90180-6

[8] Rozas, D., Law, C. and Swartzlander, G. (1997) Propagation Dynamics of Optical Vortices. Journal of the Optical Society of America B, 14, 3054-3065. https://doi.org/10.1364/JOSAB.14.003054

[9] Dennis, M.R., O’Holleran, K. and Padgett, M.J. (2009) Singular Optics: Optical Vortices and Polarization Singularities. In: Progress in Optics, Vol. 53, Elsevier, Amsterdam, 293-363. https://doi.org/10.1016/S0079-6638(08)00205-9

[10] Cheng, W. (2013) Optical Vortex Beams: Generation, Propagation and Applications. Dissertation, the University of Dayton, Dayton, OH, 1-131.

[11] Khonina, S.N., Karpeev, S.V., Alferov, S.V. and Soifer, V.A. (2015) Generation of Cylindrical Vector Beams of High Orders Using Uniaxical Crystals. Journal of $O p$ tics, 17, 065001. https://doi.org/10.1088/2040-8978/17/6/065001

[12] Khonina, S.N., Savelyev, D.A. and Kazanskiy, N.L. (2015) Vortex Phase Elements as Detectors of Polarization State. Optics Express, 23, 17845-17859.

[13] Soskin, M. and Vasnetsov, M.V. (2001) Singular Optics. In: Progress in Optics, Vol. 42, Elsevier, Amsterdam, 219-276. https://doi.org/10.1016/S0079-6638(01)80018-4

[14] Desyatnikov, A.S., Torner, L. and Kivshar, Y.S. (2005) Optical Vortices and Vortex Solitons. In: Progress in Optics, Vol. 47, Elsevier, Amsterdam, 219-319. https://doi.org/10.1016/S0079-6638(05)47006-7

[15] Brown, T.G. (2011) Unconventional Polarization States: Beam Propagation. In: Progress in Optics, Vol. 56, Elsevier, Amsterdam, 81-129. https://doi.org/10.1016/B978-0-444-53886-4.00002-2

[16] Karpeev, S.V., Khonina, S.N. and Alferov, S.V. (2015) Generation and Conversion of Mode Beams and Their Polarization States on the Basis of Diffractive Optical Element Application. Optical Engineering, 52, Article ID: 091718. https://doi.org/10.1117/1.OE.52.9.091718

[17] Sokolov, A.L. and Murashkin, V.V. (2011) Diffraction Polarization-Optical Elements with the Radial Symmetry. Optics and Spectroscopy, 111, 900-907. https://doi.org/10.1134/S0030400X11130212

[18] Khonina, S.N. and Karpeev, S.V. (2011) Generating Inhomogeneously Polarized Higher-Order Laser Beams by Use of Diffractive Optical Elements. Journal of the Optical Society of America A, 28, 2115-2123. https://doi.org/10.1364/JOSAA.28.002115

[19] Sokolov, A.L. (2017) Optical Vortices with Axisymmetric Polarization Structure. Optical Engineering, 56, Article ID: 014109. https://doi.org/10.1117/1.OE.56.1.014109

[20] Allen, L., Beijersbergen, M.W., Spreeuw, R.J. and Woerdman, J.P. (1992) Orbital Angular Momentum of Light and the Transformation of Laguerre-Gaussian Laser Modes. Physical Review A, 45, 8185-8189. https://doi.org/10.1103/PhysRevA.45.8185

[21] Barnett, S.M. and Allen, L. (1994) Orbital Angular Momentum and Nonparaxial Light-Beams. Optics Communications, 110, 670-678. https://doi.org/10.1016/0030-4018(94)90269-0

[22] Simpson, N.B., Dholakia, K. and Padgett, M.J. (1997) Mechanical Equivalence of 
Spin and Orbital Angular Momentum of Light: An Optical Spanner. Optics Letters, 22, 52-54. https://doi.org/10.1364/OL.22.000052

[23] Bass, M. (2010) Handbook of Optics: Volume 1-Geometrical and Physical Optics, Polarized Light, Components and Instruments. 3rd Edition, New York: McGraw-Hill Companies, Inc.

[24] Akent'ev, A.S., Sadovnikov, M.A., Sokolov, A.L. and Simonov, G.V. (2017) Polarization Analysis of the Beam-Steering Device of Quantum Optical Systems. Optics and Spectroscopy, 122, 1008-1014. https://doi.org/10.1134/S0030400X17060029

[25] Kotlyar, V.V., et al. (2005) Generation of Phase Singularity through Diffracting a Plane or Gaussian Beam by a Spiral Phase Plate. Journal of the Optical Society of America A, 22, 849-861. https://doi.org/10.1364/JOSAA.22.000849

[26] Khonina, S.N., Kotlyar, V.V., Shinkarev, M.V. and Soifer, V.A. (1992) The Rotor Phase Filter. Journal of Modern Optics, 39, 1147-1154. https://doi.org/10.1080/09500349214551151

[27] Sadovnikov, M.A. and Sokolov, A.L. (2009) Spatial Polarization Structure of Radiation Formed by a Retroreflector with Nonmetalized Faces. Optics and Spectroscopy, 107, 201-206. https://doi.org/10.1134/S0030400X09080062

[28] Crabtree, K. and Chipman, R. (2010). Polarization Conversion Cube-Corner Retroreflector. Applied Optics, 49, 5882-5890. https://doi.org/10.1364/AO.49.005882

[29] Sokolov, A.L. (2013) Formation of Polarization-Symmetrical Beams Using Cube-Corner Reflectors. Journal of the Optical Society of America A, 30, 1350-1357.

https://doi.org/10.1364/JOSAA.30.001350 


\section{List of Used Symbols}

\begin{tabular}{|c|c|}
\hline$\psi$ & polarization azimuth \\
\hline$\chi$ & ellipticity angle \\
\hline$\varphi$ & coordinate azimuth in a transverse plate \\
\hline$\Gamma_{P}, \Gamma_{N}$ & polarization variables, characterizing polarization state (Jones vector components ratio) \\
\hline$q$ & number of repetitions of both phase (the optical vortex order) \\
\hline$s$ & $\begin{array}{l}\text { number of repetitions of polarization state (the order of axisymmetric polarization } \\
\text { structure) in a transverse plate }\end{array}$ \\
\hline$u$ & order of spiral rotator \\
\hline$E$ & electrical vector \\
\hline$H$ & magnetic vector \\
\hline$D$ & Jones vector \\
\hline M & Jones matrix \\
\hline SR & spiral rotator \\
\hline $\mathrm{SR}_{\mathrm{P}}$ & positivespiral rotator which rotates the polarization ellipse axes counterclockwise \\
\hline $\mathrm{SR}_{\mathrm{N}}$ & negativespiral rotator which rotates the polarization ellipse axes clockwise \\
\hline SRF & spiral polar (Faraday) rotator \\
\hline SRO & spiral non-polar rotator \\
\hline CCR & cube-corner reflector \\
\hline$\lambda$ & wavelength \\
\hline$\rho$ & curvature of the wave front \\
\hline$r$ & right circular polarization \\
\hline 1 & left circular polarization \\
\hline$R$ & right-handed optical vortex \\
\hline$L$ & left-handed optical vortex \\
\hline$r R$ & right-handed optical vortex with right circular polarization \\
\hline$I L$ & left-handed optical vortex with left circular polarization \\
\hline$r L$ & left-handed optical vortex with right circular polarization \\
\hline IR & right-handed optical vortex with left circular polarization \\
\hline
\end{tabular}

